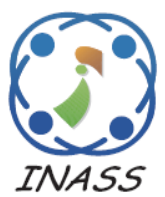

\title{
Adaptive Type-2 Fuzzy Sliding Mode Control Using Supervisory Type-2 Fuzzy Control for 6 DOF Octorotor Aircraft
}

\author{
Samir Zeghlache $^{1 *} \quad$ Mohammed Zinelaabidine Ghellab $^{1} \quad$ Abderrahmen Bouguerra $^{1}$ \\ ${ }^{1}$ Laboratoire d'Analyse des Signaux et Systèmes, Department of Electronics, Faculty of Technology, \\ University of Msila, BP 166 Ichbilia, Msila, Algeria \\ * Corresponding author's Email: zegsam5@ gmail.com
}

\begin{abstract}
Control of the helicopter includes nonlinearities, uncertainties and external perturbations that should be considered in the design of control laws. This paper presents a control strategy for 6 DOF octorotor aircraft, based on the coupling of the interval type-2 fuzzy logic control with the so-called sliding mode control (SMC) using a proportional-integral-derivative (PID) sliding surface. The main purpose is to eliminate the chattering phenomenon. For this proposed we have used an interval type-2 fuzzy logic control to generate the switching control signal. Moreover the output gain of the type-2 fuzzy sliding is tuned on-line by supervisory type-2 fuzzy system (adaptive interval type-2 fuzzy sliding mode control), so the chattering is avoided, the simulation results that are compared of conventional SMC with PID sliding surface indicate that the control performance of the 6 DOF octorotor aircraft is satisfactory and the proposed adaptive interval type-2 fuzzy sliding mode control (AIT2FSMC) can achieve favorable tracking performance.
\end{abstract}

Keywords: Type-2 Fuzzy logic system, Adaptive control, Robust control, Coaxial octorotor, Sliding mode control.

\section{Introduction}

In these last years, a growing interest has been shown in robotics. In fact, several industries (automotive, medical, manufacturing, space, ....), require robots to replace men in dangerous, boring or onerous situations. A wide area of this research is dedicated to aerial platform. The multi rotors aerial platform are an underactuated systems and the states are highly coupled. Great efforts have been made to control multi rotors helicopter and some strategies have been developed to solve the path following problems for this type of system. In [1] presents a PID controller for a stabilization of a quadrotor UAV. This research successfully demonstrated through experiments that a simple PID control is sufficient for stable flight. In [2] introduces a particle swarm optimization (PSO) method to adjust the PID parameters. PID and linear quadratic regulator (LQR) trajectory tracking control are designed in [3] for an unmanned quadrotor helicopter. On the other hand, the inconvenient of this method is suit for the linear system and it is not convenient for nonlinear systems except making some hypothesis.

The sliding mode control has been tested extensively to control quadrotors. The advantage of this approach is its insensitivity to the model errors and parametric uncertainties, as well as the ability to globally stabilize the system in the presence of other disturbances [4]. In [5, 6-7] a sliding mode control has been used to control a quadrotor helicopter. In [8] the authors present a continuous sliding mode control method based on feedback linearization applied to the quadrotor. A controller based on backstepping and sliding mode techniques for miniature quadrotor helicopter is presented in $[9,10$ 11]. The major drawback is the phenomenon of chattering that existing in the control signals caused by the discontinuous control in the proposed method. In $[12,13]$ a second order sliding mode control is proposed to control the autonomous helicopter this control technique is able to eliminate the chattering, but it is very sensitive to noise and needs the 
information about the sliding variable and its derivatives, which are not always available. The proposed method not needs to know the derivatives of the sliding variable.

In this paper, a control algorithm is developed by combining the interval type-2 fuzzy approach with the PID sliding mode control method. This contribution bases on the combination of the adaptive interval type-2 fuzzy algorithm and robust control technique in order to eliminate the chattering phenomenon for uncertain aeronautic system. We present a control technique based on the development and the synthesis of a control algorithm which bases upon sliding mode by using the PID sliding surfaces and ensures the locally asymptotic stability and desired tracking trajectories expressed in term of the centre of mass coordinates along $(x, y, z)$ axis and yaw angle, while the desired roll and pitch angles are deduced unlike to [14]. Finally all synthesized control laws are highlighted by simulations providing satisfactory results. A quantitative comparison confirmed that the performance of proposed adaptive type-2 fuzzy sliding mode control with PID sliding surfaces is better than a conventional sliding mode controller with PID-sliding surfaces.

The rest of the paper is organized as follows. Section 2 focuses on the nonlinear dynamic model of the coaxial octorotor. In section 3, problem formulation is presented. Interval type-2 fuzzy sliding mode controller (IT2FSMC) design is presented in section 4. Section 5 presents the adaptive type-2 fuzzy sliding mode controller (AIT2FSMC). Simulation results and related discussions are given in section 6. Finally some conclusions are drawn in section 7.

\section{Dynamical Modeling of the coaxial octorotor}

The configuration of the octorotor is represented in Fig. 1. It is similar to a quadrotor with two coaxial counter-rotating motors at the ends of each arm. This configuration has several advantages compared to the star configuration used in the literature $[15,16]$ in terms of stability and size. A classical star octorotor needs more arms, and each arm needs to be longer to guarantee adequate spacing among the rotors. We have adopted this configuration for its higher thrust to weight ratio.

Consider first a body-fixed reference frame RB with the $X, Y, Z$ axis originating at the center of mass of the vehicle.

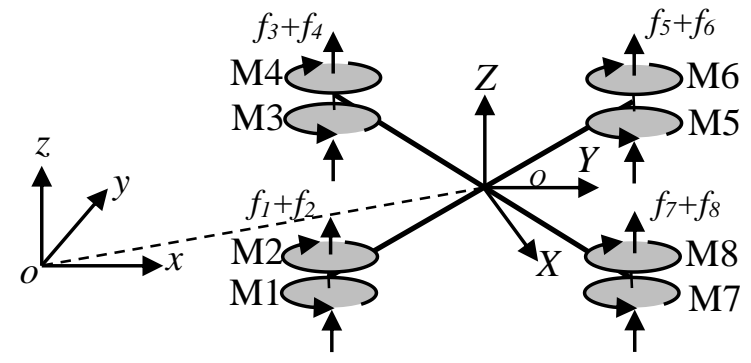

Figure.1 Coaxial octorotor configuration.

The $\mathrm{X}$ axis points to the front direction, the $\mathrm{Y}$ axis to the left direction and the $\mathrm{Z}$ axis upwards. Consider second an inertial frame RI fixed to the earth $\{0, x, y, z\}$. The equations governing the motion of the system are obtained using the EulerLagrange approach and give the commonly used model [17]

$$
\left\{\begin{array}{l}
\ddot{z}=\frac{\cos \varphi \cos \theta}{m} u_{1}-g-\frac{K_{1}}{m} \dot{z}+F_{d 1} \\
\ddot{x}=u_{2}-\frac{K_{2}}{m} \dot{x}+F_{d 2} \\
\ddot{y}=u_{3}-\frac{K_{3}}{m} \dot{y}+F_{d 3} \\
\ddot{\varphi}=\left(\frac{I_{y}-I_{z}}{I_{x}}\right) \dot{\theta} \dot{\psi}-\frac{K_{4}}{I_{x}} \dot{\varphi}^{2}-\frac{J_{H} \bar{\Omega}}{I_{x}} \dot{\theta}+\frac{1}{I_{x}} u_{4}+M_{d 1} \\
\ddot{\theta}=\left(\frac{I_{z}-I_{x}}{I_{y}}\right) \dot{\varphi} \dot{\psi}-\frac{K_{5}}{I_{y}} \dot{\theta}^{2}-\frac{J_{H} \bar{\Omega}}{I_{y}} \dot{\varphi}+\frac{1}{I_{y}} u_{5}+M_{d 2} \\
\ddot{\psi}=\left(\frac{I_{x}-I_{y}}{I_{z}}\right) \dot{\varphi} \dot{\theta}-\frac{K_{6}}{I_{z}} \dot{\psi}^{2}+\frac{1}{I_{z}} u_{6}+M_{d 3}
\end{array}\right.
$$

Where:

$$
\left\{\begin{array}{l}
u_{2}=\frac{u_{1}}{m}(\cos \varphi \sin \theta \cos \psi+\sin \varphi \sin \psi) \\
u_{3}=\frac{u_{1}}{m}(\cos \varphi \sin \theta \sin \psi-\sin \varphi \cos \psi)
\end{array}\right.
$$

Where $m$ denotes the total mass, g represents the acceleration of gravity, $l$ denotes the distance from the center of each rotor to the center of gravity, $K_{1}, \ldots, K_{6}$ denote the drag coefficients and positive constants, $d_{1}, \ldots, d_{6}$ are the effect of external disturbances that affect the movement of the octorotor helicopter are produced by unknown wind gust.

$\bar{\Omega}=\Omega_{2}+\Omega_{3}+\Omega_{6}+\Omega_{7}-\Omega_{1}-\Omega_{4}-\Omega_{5}-\Omega_{8}$

$\Omega_{i}$; stand for the angular speed of the propeller $i$ with $i=i_{1}, \ldots, i_{8}$. 
$I_{x}, I_{y}, I_{z}$ represent the inertias of the coaxial octorotor; $J_{H}$ denotes the inertia of the propeller; $u_{1}$ denotes the total thrust on the body in the z-axis; $u_{4}$ and $u_{5}$ represent the roll and pitch inputs, respectively; $u_{6}$ denotes a yawing moment.

$$
\left\{\begin{array}{l}
u_{1}=F_{1}+F_{2}+F_{3}+F_{4}+F_{5}+F_{6}+F_{7}+F_{8} \\
u_{4}=l \frac{\sqrt{2}}{2}\left(F_{7}+F_{8}+F_{5}+F_{6}-F_{3}-F_{4}-F_{1}-F_{2}\right) \\
u_{5}=l \frac{\sqrt{2}}{2}\left(F_{3}+F_{4}+F_{5}+F_{6}-F_{7}-F_{8}-F_{1}-F_{2}\right) \\
u_{6}=\frac{d}{b}\left(F_{2}+F_{3}+F_{6}+F_{7}-F_{1}-F_{4}-F_{5}-F_{8}\right)
\end{array}\right.
$$

Where $F_{i}=b \Omega^{2}{ }_{i}$ with $i=i_{1}, \ldots, i_{8}$ denote the thrust generated by eight rotors and are considered as the real control inputs to the dynamical system, $b$ denotes the lift coefficient; $d$ denotes the force to moment scaling factor.

The input vector is given by:

$u=\left[\begin{array}{llllll}u_{1} & u_{2} & u_{3} & u_{4} & u_{5} & u_{6}\end{array}\right]^{T}$

The output vector is given by:

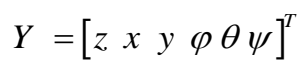

The lumped disturbance vectors acting on the aircraft is given by:

$$
F_{d}=\left[\begin{array}{lll}
F_{d 1} & F_{d 2} & F_{d 3}
\end{array}\right]^{T}, M_{d}=\left[\begin{array}{lll}
M_{d 1} & M_{d 2} & M_{d 3}
\end{array}\right]^{T}
$$

\section{Assumption 1:}

The roll, the pitch and the yaw angles $(\varphi, \theta, \psi)$ are bounded as follows: roll angle by $-\pi / 2<\varphi<\pi / 2$; pitch angle, $-\pi / 2<\theta<\pi / 2$; and yaw angle, $-\pi<\psi<\pi$.

The model (1) developed in the first part of this paper can be rewritten in the following form:

$$
\ddot{X}=A \dot{X}+E g(X)+D(t)+F(X) u(t)
$$

Where:

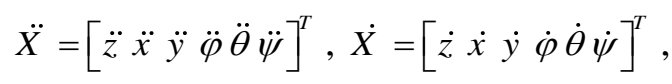

$$
A=\left[\begin{array}{cccccc}
-\frac{K_{1}}{m} & 0 & 0 & 0 & 0 & 0 \\
0 & -\frac{K_{2}}{m} & 0 & 0 & 0 & 0 \\
0 & 0 & -\frac{K_{3}}{m} & 0 & 0 & 0 \\
0 & 0 & 0 & 0 & -\frac{J_{H} \bar{\Omega}}{I_{x}} & 0 \\
0 & 0 & 0 & -\frac{J_{H} \bar{\Omega}}{I_{x}} & 0 & 0 \\
0 & 0 & 0 & 0 & 0 & 0
\end{array}\right],
$$

$$
E=[111111]^{T}, g(X)=\left[\begin{array}{c}
-g \\
0 \\
0 \\
\left(\frac{I_{y}-I_{z}}{I_{x}}\right) \dot{\theta} \dot{\psi}-\frac{K_{4}}{I_{x}} \dot{\varphi}^{2} \\
\left(\frac{I_{z}-I_{x}}{I_{y}}\right) \dot{\varphi} \dot{\psi}-\frac{K_{5}}{I_{y}} \dot{\theta}^{2} \\
\left(\frac{I_{x}-I_{y}}{I_{z}}\right) \dot{\varphi} \dot{\theta}-\frac{K_{6}}{I_{z}} \dot{\psi}^{2}
\end{array}\right]
$$

$$
D(t)=\left[\begin{array}{lllll}
F_{d 1} & F_{d 2} & F_{d 3} & M_{d 1} & M_{d 2} \\
M_{d 3}
\end{array}\right]^{T},
$$

$$
F(X)=\left[\begin{array}{cccccc}
\frac{\cos \varphi \cos \theta}{m} & 0 & 0 & 0 & 0 & 0 \\
0 & 1 & 0 & 0 & 0 & 0 \\
0 & 0 & 1 & 0 & 0 & 0 \\
0 & 0 & 0 & \frac{1}{I_{x}} & 0 & 0 \\
0 & 0 & 0 & 0 & \frac{1}{I_{y}} & 0 \\
0 & 0 & 0 & 0 & 0 & \frac{1}{I_{z}}
\end{array}\right]
$$

If some uncertainties representing parameter variations, $\Delta A, \Delta E$ and $\Delta F(X)$ are assumed to be present on the system, Eq. (7) can be rearranged as:

$$
\begin{aligned}
\ddot{X}= & (A+\Delta A) \dot{X}+(E+\Delta E) g(X)+D(t)+ \\
& (F(X)+\Delta F(X)) u(t)
\end{aligned}
$$

Assumption 2 [18]:

The uncertainties are bounded such that $\triangle A l \leq 1$ $\Delta A|\leq \Delta A h, \Delta E l \leq| \Delta E|\leq \Delta E h, \Delta F l(X) \leq| \Delta F(X) \mid \leq$ $\triangle F h(X)$ and the subscripts 1 and $\mathrm{h}$ denote lower and upper uncertainty values. 


\section{Problem formulation}

To achieve a robust path following for the octorotor helicopter, two techniques, capable of controlling the helicopter in presence of sustained external disturbances, parametric uncertainties and unmodelled dynamics, are combined. The proposed control strategy is based on the decentralized structure of the octorotor helicopter system, which is composed of the dynamic equation (1).

The translational motion control is performed in two stages. In the first one, the helicopter height, $z$, is controlled and the total thrust, u1, is the manipulated signal. In the second stage, the reference of pitch and roll angles $\left(\theta_{d}\right.$ and $\varphi_{d}$ respectively) are generated through the two virtual inputs $u_{2}$ and $u_{3}$, computed to follow the desired $x y$ movement. Finally the rotation controller is used to stabilize the octorotor under near quasi-stationary conditions with control inputs $u_{4}, u_{5}, u_{6}$.

The desired roll and pitch angles in terms of errors between actual and desired speeds are, thus, separately given by:

$$
\begin{aligned}
& \varphi_{d}=\arcsin \left(\frac{m u_{2}}{u_{1}} \sin \psi-\frac{m u_{3}}{u_{1}} \cos \psi\right) \\
& \theta_{d}=\arcsin \left(\frac{m u_{2}}{u_{1}(\cos \varphi \cos \psi)}-\frac{\sin \varphi \sin \psi}{\cos \varphi \cos \psi}\right)
\end{aligned}
$$

Fig. 2 shows the actuated octorotor helicopter with six inputs $u_{1}, \ldots, u_{6}$ and six outputs $(z, x, y, \varphi, \theta, \psi)$.

The main objective of the control design is to develop an adaptive type-2 fuzzy sliding mode tracking controller able to force the system output $(x(t), y(t), z(t), \psi(t))$ to follow the given reference signal,

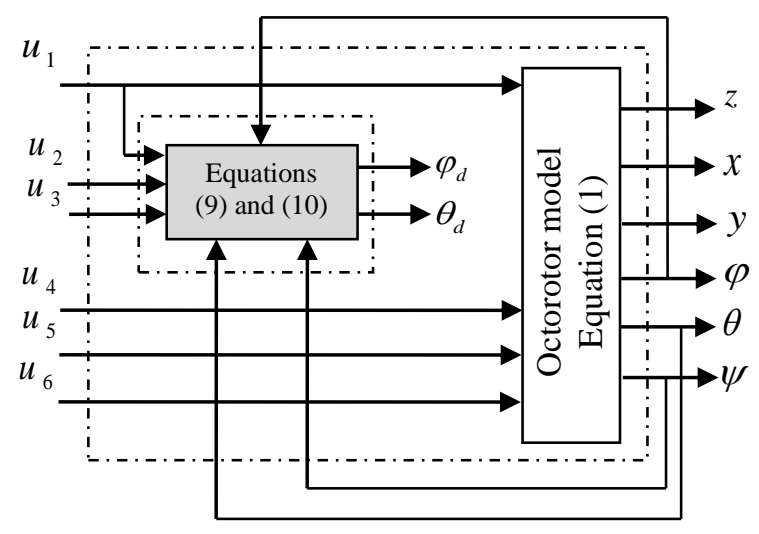

Figure.2 Block diagram of the control structure of the octorotor helicopter. $\left(x_{d}(\mathrm{t}), y_{d}(\mathrm{t}), z_{d}(\mathrm{t}), \psi_{d}(t)\right)$ under plant uncertainties, external disturbances, while ensuring that all involved signals in the closed-loop system remain bounded.

\section{Type-2 fuzzy sliding mode controller design}

It is known that the crucial and the most important step of sliding mode control (SMC) design is the construction of the sliding surface $s(t)$ which is expected to response desired control specifications and performance [18]. The trajectories are enforced to lie on the sliding surfaces. The PID sliding surface in the space of tracking error can be defined as [18]:

$s(t)=K_{p} e(t)+K_{i} \int_{0}^{t} e(\xi) d \xi+K_{d} \frac{d}{d t} e(t)$

Where $K_{p}$ is $n^{*} n$ positive proportional gain matrix, $K_{i}$ is $\mathrm{n}^{*} \mathrm{n}$ positive integral gain matrix, and $K_{d}$ is $n^{*} n$ positive derivative gain matrix parameters to be selected. For 6 DOF octorotor aircraft, $n=6$ and $, K_{p}=\operatorname{diag}\left\{k_{p 1}, k_{p 2}, k_{p 3}, k_{p 5}, k_{p 6}\right\}, K_{d}=\operatorname{diag}\left\{k_{d 1}\right.$, $\left.k_{d 2}, k_{d 3}, k_{d 5}, k_{d 6}\right\}$ and $K_{i}=\operatorname{diag}\left\{k_{i l}, k_{i 2}, k_{i 3}, k_{i 5}, k_{i 6}\right\}$ and $e(t)=Y_{d}(\mathrm{t})-Y(t)$ is the tracking position error, in which $Y_{d}(t)=\left[\begin{array}{llllll}z_{d} & x_{d} & y_{d} & \varphi_{d} & \theta_{d} & \psi_{d}\end{array}\right]^{T}$ is the desired trajectory. In the case of present work; in order to eliminate chattering phenomenon, a continuous type-2 fuzzy logic control is used to approximate the switching control. The configuration of the proposed interval type-2 sliding mode control (IT2FSMC) scheme is shown in Fig. 3; it contains an equivalent control part and a two inputs single output interval type-2 fuzzy logic.

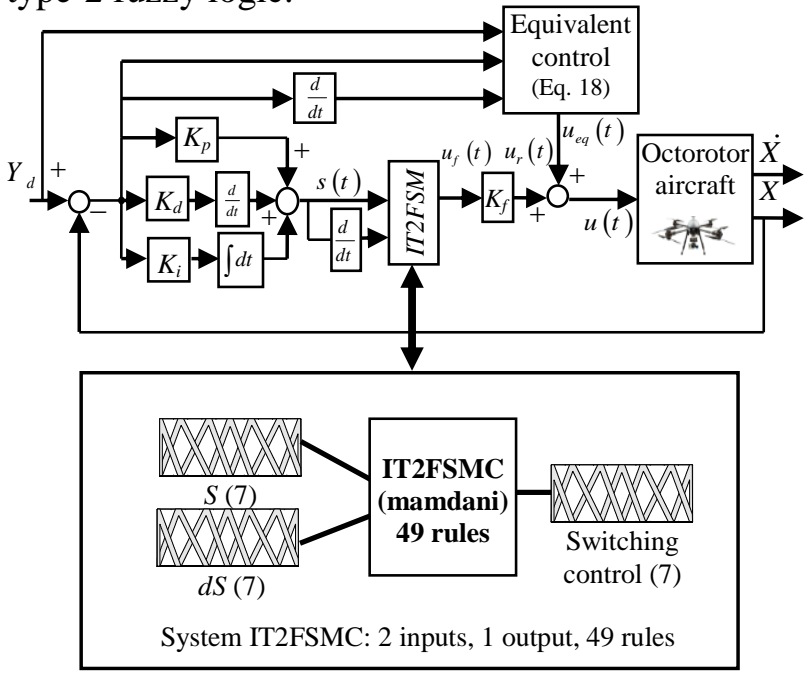

Figure.3 Block diagram of the IT2FSMC with PID sliding surface. 
The discontinuous control is computed by:

$u_{r}(t)=K_{f} u_{f}(t)$

With:

$$
u_{f}(t)=\operatorname{IT} 2 F S M C(s, \dot{s})
$$

Where $K_{f}$ is the normalization factor of the output variable, and $u_{f}(t)$ is the output of the IT2FSMC, which is obtained by the normalized $s(t)$ and its derivative. The fuzzy type- 2 membership functions of the input linguistic variables $s(t)$ and its derivative, and the output switching control $u_{f}(t)$ are presented by Fig. 4. The fuzzy control surface of the output $u_{f}(t)$ is shown in Fig. 5. The simulation model of different membership functions such as triangular, trapezoidal, gaussian and bell shaped functions in IT2FSMC of the coaxial octorotor is implemented in Matlab/simulink the results of the simulated performances are compared. The triangular membership function shows better performance compared to other membership functions.

In order to attenuate the chattering effect and handle the uncertainty of the eight rotors helicopter, a type-2 fuzzy controller has been used with two inputs and single output for each subsystem.
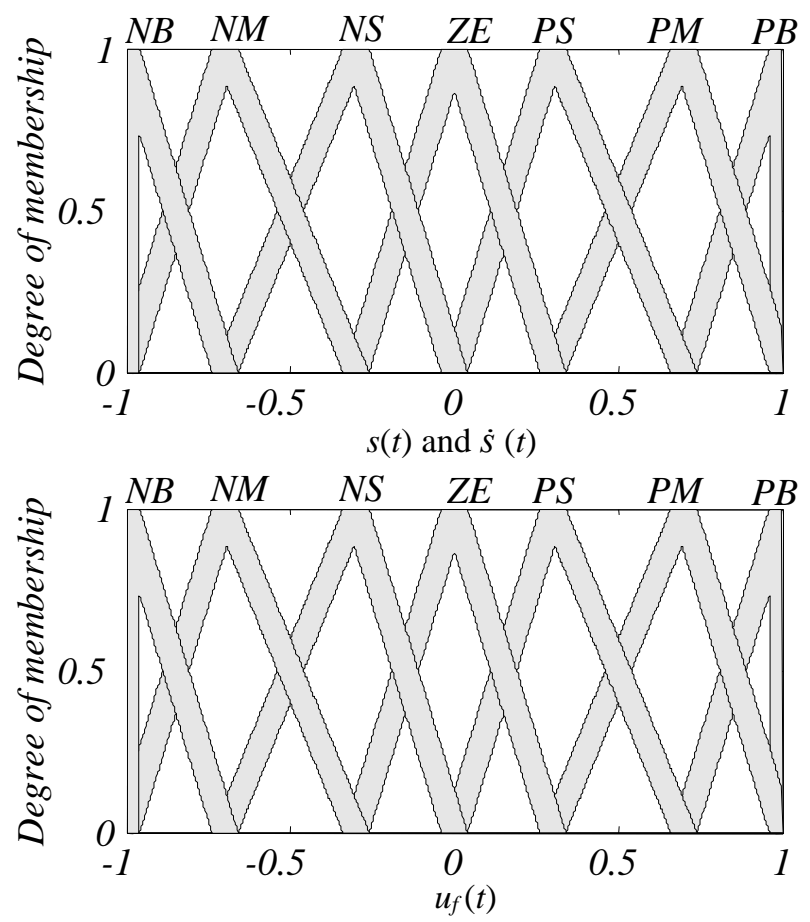

Figure.4 Membership functions of the inputs $\mathrm{s}(t)$ and $\dot{s}(t)$ and output $u_{f}(t)$

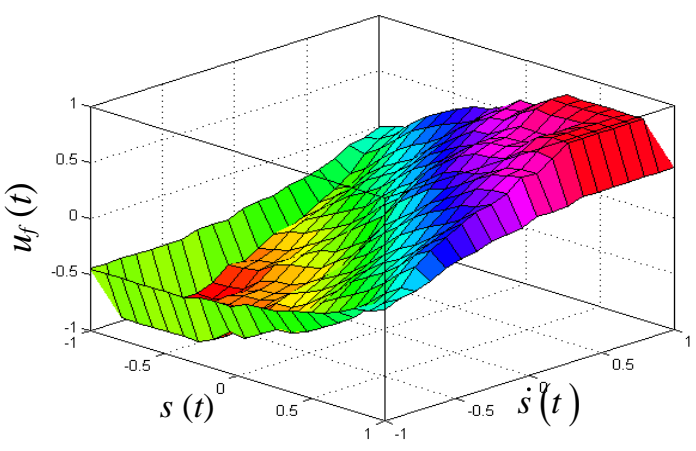

Figure.5 Control surface of $u_{f}(t)$.

Table 1. Rule matrix of IT2FSMC [18]

\begin{tabular}{|c|c|c|c|c|c|c|c|}
\hline $\boldsymbol{u}_{\boldsymbol{f}}(\boldsymbol{t})$ & \multicolumn{7}{|c|}{$\boldsymbol{s}(\boldsymbol{t})$} \\
\hline$\dot{\boldsymbol{s}}(\boldsymbol{t})$ & $N B$ & $N M$ & $N S$ & $Z E$ & $P S$ & $P M$ & $P B$ \\
\hline$N B$ & $N B$ & $N B$ & $N B$ & $N B$ & $N M$ & $N S$ & $N B$ \\
\hline$N M$ & $N B$ & $N B$ & $N B$ & $N M$ & $N S$ & $Z E$ & $P S$ \\
\hline$N S$ & $N B$ & $N B$ & $N M$ & $N S$ & $Z E$ & $P S$ & $P M$ \\
\hline$Z E$ & $N B$ & $N M$ & $N S$ & $Z E$ & $P S$ & $P M$ & $P B$ \\
\hline$P S$ & $N M$ & $N S$ & $Z E$ & $P S$ & $P M$ & $P B$ & $P B$ \\
\hline$P M$ & $N S$ & $Z E$ & $P S$ & $P M$ & $P B$ & $P B$ & $P B$ \\
\hline$P B$ & $Z E$ & $P S$ & $P M$ & $P B$ & $P B$ & $P B$ & $P B$ \\
\hline
\end{tabular}

Then, the inputs of the controllers are the sliding surface and its derivative and the output is the switching control. $u_{f}(t)$ All the membership functions of the fuzzy inputs variable are chosen to be triangular for all upper and lower membership functions. The used labels of the fuzzy variable $s(t)$ and its derivative are: negative big $(N B)$, negative medium $(N M)$, negative small $(N S)$, zero $(Z E)$, positive big $(P B)$, positive medium $(P M)$, positive small $(P S)\}$. The switching control is decomposed into seven levels represented by a set of linguistic variables: \{negative big $(N B)$, negative medium $(N M)$, negative small $(N S)$, zero $(Z E)$, positive big $(P B)$, positive medium $(P M)$, positive small $(P S)\}$. Table 1 presents the rules base which contains 49 rules;

The control law is computed by:

$$
\begin{aligned}
u(t) & =u_{e q}(t)+u_{r}(t)=u_{e q}(t)+K_{f} u_{f}(t) \\
& =u_{e q}(t)+K_{f} \operatorname{IT} 2 \operatorname{FSMC}(s, \dot{s})
\end{aligned}
$$

Stability analysis:

$$
\begin{aligned}
& V(t)=\frac{1}{2} s^{T}(t) s(t) \\
& \dot{V}(t)=s^{T}(t) \dot{s}(t)
\end{aligned}
$$

Eq. (8) can be rewritten as: 
$\ddot{X}=A \dot{X}+E g(X)+F(X) u(t)+L(t)$

Where:

$$
L(t)=\Delta A \dot{X}+\Delta E g(X)+D(t)+\Delta F(X) u(t)
$$

The time derivative of the sliding surface determined by (11) is obtained by:

$$
\begin{aligned}
\dot{s}(t)= & K_{p} \dot{e}(t)+K_{i} e(t)+K_{d}\left[\ddot{Y}_{d}-A \dot{X}-E g(X)\right. \\
& -L(t)-F(X) u(t)]
\end{aligned}
$$

The control law being derived as the solution of $\dot{s}(\mathrm{t})=0$ without considering uncertainty $(L(t)=0)$ is to achieve the desired performance under nominal model, and it is referred to as equivalent control law $[18,19-20]$, represented by $u_{e q}(t)$.

$$
\begin{aligned}
u_{e q}(t)= & \left(K_{d} F(X)\right)^{-1}\left[K_{p} \dot{e}(t)+K_{i} e(t)+K_{d} \ddot{Y_{d}}-\right. \\
& \left.K_{d} A \dot{X}-K_{d} E g(X)\right]
\end{aligned}
$$

The time derivative of Lyapunov function in Eq. (15), is obtained by:

$$
\dot{V}(t)=s^{T}(t) \dot{s}(t)=s^{T}\left\{K_{p} \dot{e}+K_{i} e+K_{d}\left[\ddot{Y}_{d}-\ddot{X}\right]\right\}
$$

Substituting the Eq. (16) in the Eq. (55), yields

$$
\begin{aligned}
\dot{V}(t)= & s^{T}\left\{K_{p} \dot{e}+K_{i} e+K_{d}\left[\ddot{Y}_{d}-A \dot{X}-E g(X)-\right.\right. \\
& \left.\left.F(X)\left(u_{e q}(t)+u_{r}(t)\right)-L(t)\right]\right\}
\end{aligned}
$$

Substituting the equivalent control from Eq. (18), Eq. (20) becomes:

$$
\dot{V}(t)=s^{T}\left\{-\left(K_{d} F(X)\right) u_{r}(t)-K_{d} L(t)\right\}
$$

Substituting the Eq. (12) in the Eq. (21), yields

$$
\dot{V}(t)=s^{T}\left\{-\left(K_{d} F(X)\right) K_{f} \operatorname{IT} 2 F S M C(s, \dot{s})-K_{d} L(t)\right\}
$$

The output type- 2 fuzzy sets are normalized in the interval $(-1,1)$, then:

$$
\begin{aligned}
& \left|u_{f}=I T 2 \operatorname{FSMC}(s, \dot{s})\right| \leq 1 \\
& \&\left|s^{T}\right|\left|u_{f}(t)\right|=|s||\operatorname{IT} 2 \operatorname{FSMC}(s, \dot{s})| \leq|s|
\end{aligned}
$$

$$
\begin{aligned}
\dot{V}(t) & =-\left(K_{d} F(X)\right) K_{f}|s|-K_{d}|L(t)||s| \\
& =-\left[\left(K_{d} F(X)\right) K_{f}+K_{d}|L(t)|\right]|s|
\end{aligned}
$$

If we select $\left(K_{d} F(X)\right) K_{f}+K_{d}|L(t)|>0$, we can conclude that the sliding condition $s^{T}(t) \dot{s}(t)<0$ is satisfied. Thus the closed loop system is asymptotically stable and the error state trajectory converges to the sliding surface $s(t)=0$.

\section{Adaptive type-2 fuzzy sliding mode controller}

The supervisory type-2 fuzzy of the proposed tuning method contains operators knowledge in the form IF-THEN rules decide the control gains $K_{f}$ according to the current operating condition of the controlled system. Here, the control rules of the supervisory fuzzy system are developed with the error $e$ and derivative of error $\dot{e}$ as the premise. And $K_{f}=\operatorname{diag}\left\{k_{f 1}, k_{f 2}, k_{f 3}, k_{f 5}, k_{f 6}\right\}$ as a consequent of each rules [18]. The general structure of the proposed controller is given in Fig. 6. The membership functions for the inputs and outputs as shown in Fig. 7 , Fig. 8 shows the type- 2 fuzzy control surface of $K_{f}$. This surface has been used to adaptively tune $K f$ $=\operatorname{diag}\left\{k_{f 1}, k_{f 2}, k_{f 3}, k_{f 5}, k_{f 6}\right\}$ on line. The physical domain of the inputs $(e, \dot{e})$ is in the range $\{-0.01$, $0.01\}$ and that of the output $K_{f}$ is in the range $\{-0.2$, 2 \}, respectively, selected based on trial and error approach. The fuzzy variables are defined for the rule base as, $(e, \dot{e})=\{N B$ (Negative Big), $N M$ (Negative Medium), NS (Negative Small), $Z E$ (Zero), PS (Positive Small), PM (Positive Medium), $P B$ (Positive Big) $\} ; K_{f}=\{V V S$ (Very Very Small), $V S$ (Very Small), S (Small), M (Medium), $B$ (Big), $V B$ (Very Big) and $V V B$ (Very Very Big). the linguistic fuzzy rules of the supervisory type-2 fuzzy system are given in Table 2 .

Table 2. Rule matrix of supervisory type-2 fuzzy control [18]

\begin{tabular}{|c|c|c|c|c|c|c|c|}
\hline $\boldsymbol{K}_{\boldsymbol{f}}$ & \multicolumn{7}{|c|}{$\boldsymbol{e}(\boldsymbol{t})$} \\
\hline$\dot{\boldsymbol{e}}(\boldsymbol{t})$ & $N B$ & $N M$ & $N S$ & $Z E$ & $P S$ & $P M$ & $P B$ \\
\hline$N B$ & $M$ & $S$ & $V S$ & $V V S$ & $V S$ & $S$ & $M$ \\
\hline$N M$ & $B$ & $M$ & $S$ & $V S$ & $S$ & $M$ & $B$ \\
\hline$N S$ & $V B$ & $B$ & $M$ & $S$ & $M$ & $B$ & $V B$ \\
\hline$Z E$ & $V V B$ & $V B$ & $B$ & $M$ & $B$ & $V B$ & $V V B$ \\
\hline$P S$ & $V B$ & $B$ & $M$ & $S$ & $M$ & $B$ & $V B$ \\
\hline$P M$ & $B$ & $M$ & $S$ & $V S$ & $S$ & $M$ & $B$ \\
\hline$P B$ & $M$ & $S$ & $V S$ & $V V S$ & $V S$ & $S$ & $M$ \\
\hline
\end{tabular}

Therefore, Eq. (22) becomes: 


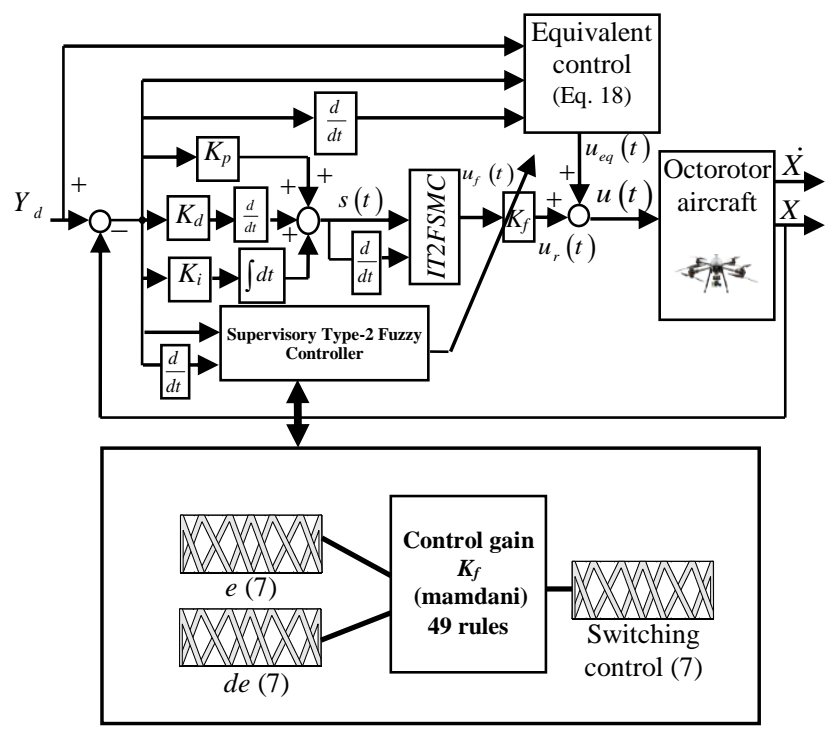

Figure.6 Block diagram of the adaptive fuzzy sliding mode control with PID sliding surface.
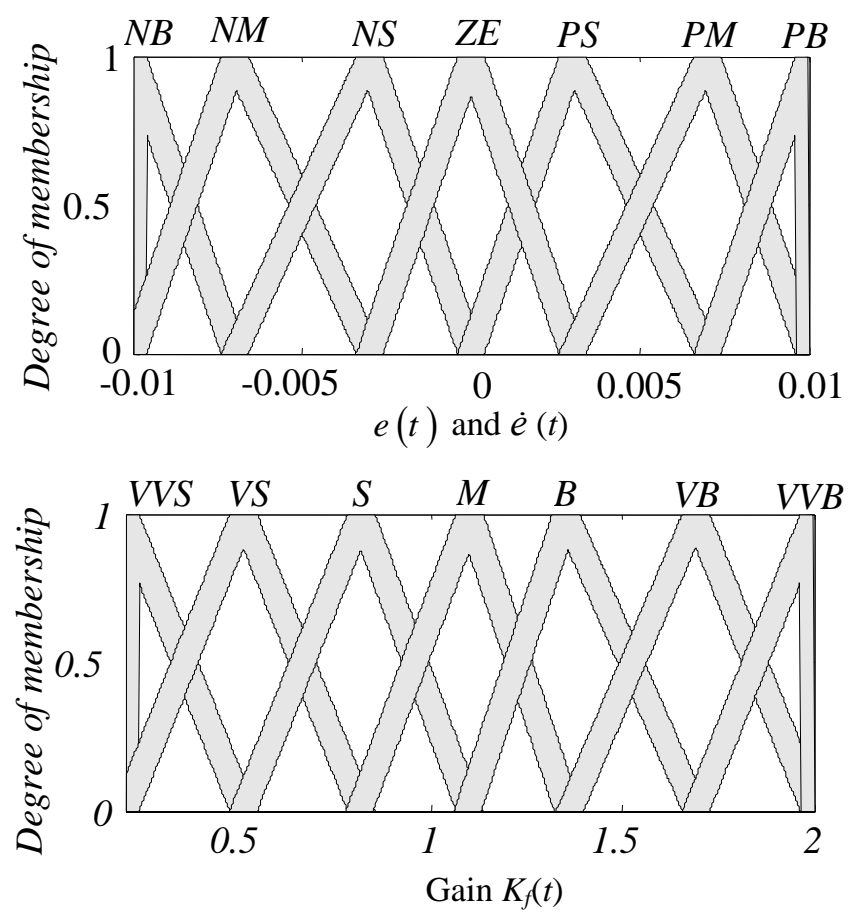

Figure.7 Membership functions of the inputs $(e(t), \dot{e}(\mathrm{t}))$ and output $K_{f}$.

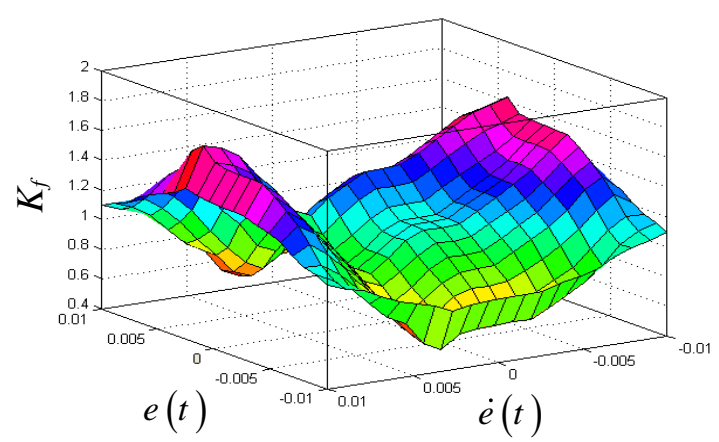

Figure. 8 Type-2 fuzzy control surfaces of $K_{f}$.

\section{Simulation results}

The proposed control strategy has been tested by simulation in order to check the effectiveness and the performance attained for the path following problem. The parameters of octorotor system are given as [14]:

$m=1.6 \mathrm{~kg}, l=0.23 \mathrm{~m}$, the nominal values of $J$ are $J_{0}=$ $\left(I_{x}, I_{y}, I_{z}\right)=\operatorname{diag}\left(4.2 \times 10^{-2}, 4.2 \times 10^{-2}, 7.5 \times 10^{-2}\right) \mathrm{Kg} . \mathrm{m}^{2}$, $b=3 \times 10-5{N s^{2}}^{2} d=7 \times 10-7 \quad \mathrm{Nms}^{2}, J_{H}=2.8385 \times 10^{-5}$ $K g . m^{2}, \quad K_{1}=K_{2}=5.5670 \times 10^{-4} \mathrm{~N} / \mathrm{m} / \mathrm{s}$, $\mathrm{K} 3=6.3540 \times 10^{-4} \mathrm{~N} / \mathrm{m} / \mathrm{s}, K_{4}=K_{5}=5.5670 \times 10^{-4}$ $\mathrm{N} / \mathrm{rad} / \mathrm{s}, K_{6}=6.3540 \times 10-4 \mathrm{~N} / \mathrm{rad} / \mathrm{s}, \mathrm{g}=9.8 \mathrm{~m} / \mathrm{s}^{2}$.

The dynamical model of coaxial octorotor with the proposed control techniques is simulated on Matlab/Simulink by using Euler method with step $\mathrm{h}=0.01$. The initial position and angle values of the octorotor for simulation tests are: $[\varphi(0)=0, \theta(0)=0$, $\psi(0)=0]$ and $[x(0)=0, y(0)=0, z(0)=0]$.

The parameters of the PID sliding surface are set as: $K_{p}=\operatorname{diag}\{1.5,1.5,1.5,1.5,1.5\}, K_{i}=\operatorname{diag}\{2,2,2$, $2,2\}$ and $K_{d}=\operatorname{diag}\{1,1,1,1,1\}$. For the IT2FSMC, the hitting control gain $K_{f}$ is set as: $K_{f}=\operatorname{diag}\{0.8,0.8$, $0.8,0.8,0.8\}$ For the AIT2FSMC, the range of the output gain $K_{f}=\operatorname{diag}\left\{k_{f 1}, k_{f 2}, k_{f 3}, k_{f 5}, k_{f 6}\right\}$ is $(0.2,2)$. Fig. 9 shows the absolute position of the octorotor in 3D, Fig. 10 shows the three positions $(x, y$, and $z)$ and the three angles (yaw, roll, and pitch).

The values of different errors for the positions and angles are tabulated in Table 3. From the simulation results, it is concluded that our proposed AIT2FSMC showed superior performance for each of the three positions and three angles For the AIT2FSMC, it is observed that both (IAE) and (ITAE), for the three positions and three angles are considerably reduced in magnitude than the other conventional method dealt with in this paper. Simulation results show that the proposed AIT2FSMC has faster tracking with smaller error values than both conventional SMC and IT2FSMC. It is observed that the proposed AIT2FSMC has the smallest IAE and ITAE performance indices among the other controllers, which proves the efficiency of the proposed controller.

On the other hand, it can be seen from the control curvature presented in Fig. 11 the conventional SMC and IT2FSMC produces a serious chattering phenomenon, On the contrary the charting phenomenon of the controlled system was suppressed in the proposed IT2FSMC controller. 


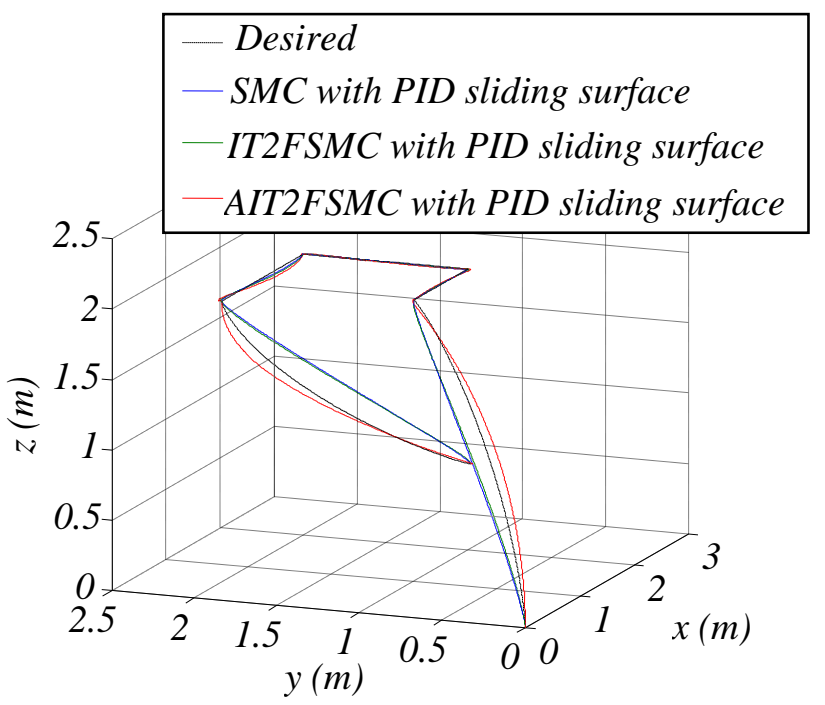

Figure.9 Absolute position of the octorotor.

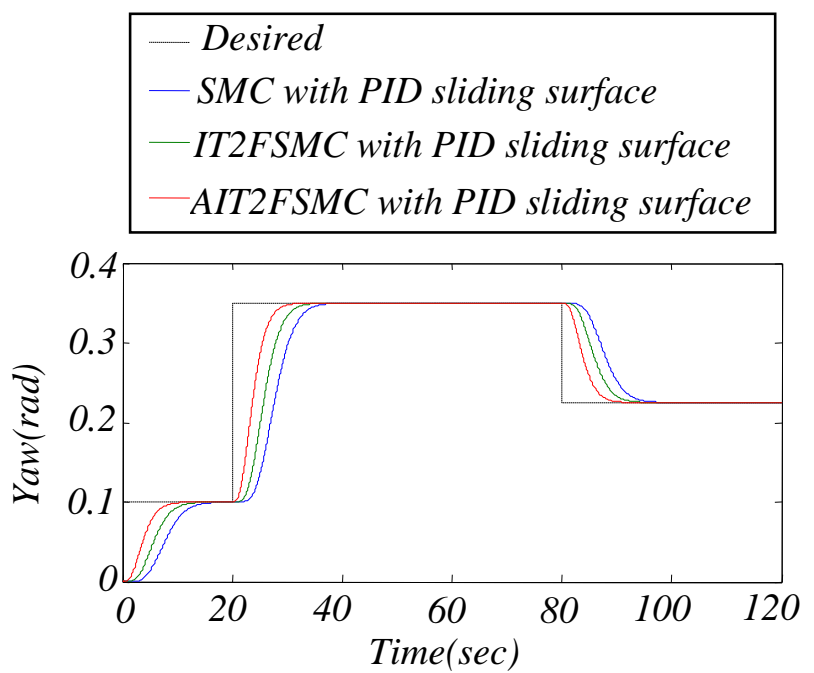

(a)

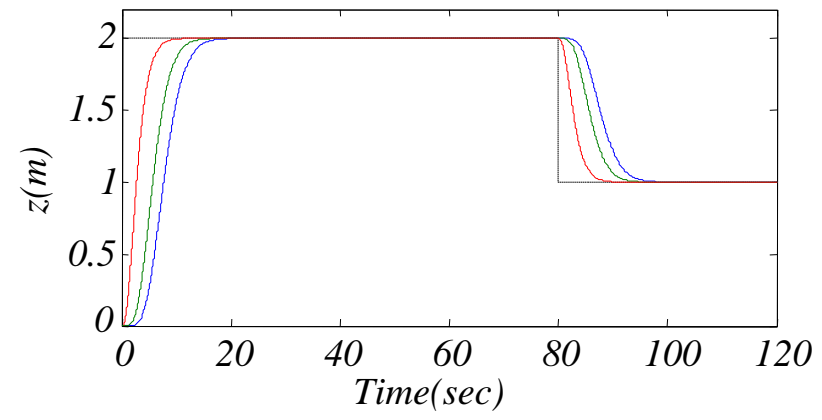

(b)

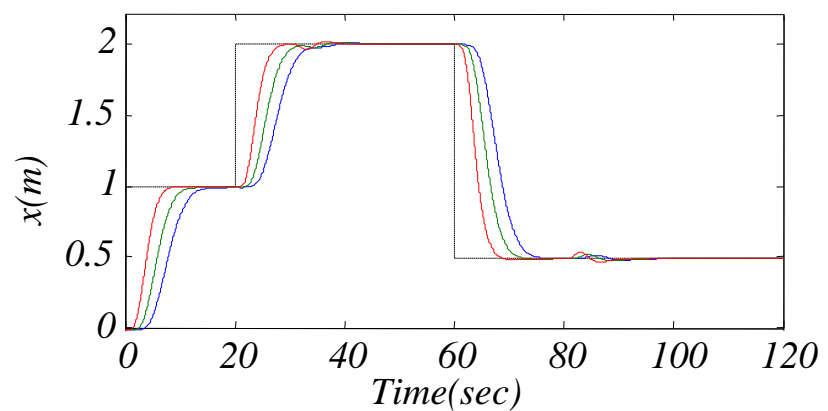

(c)

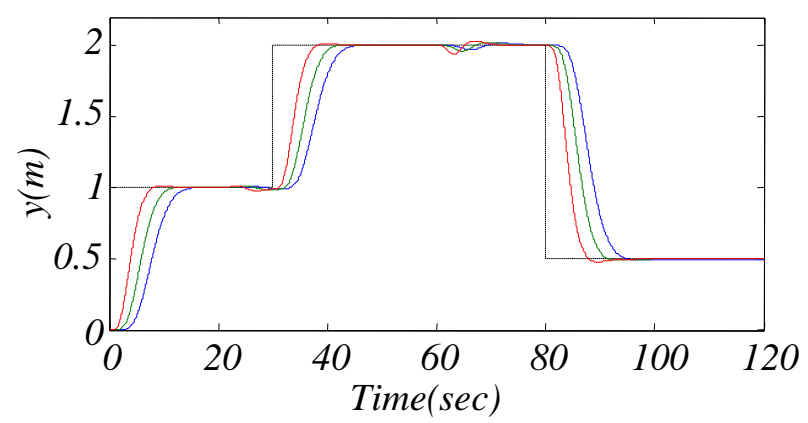

(d)

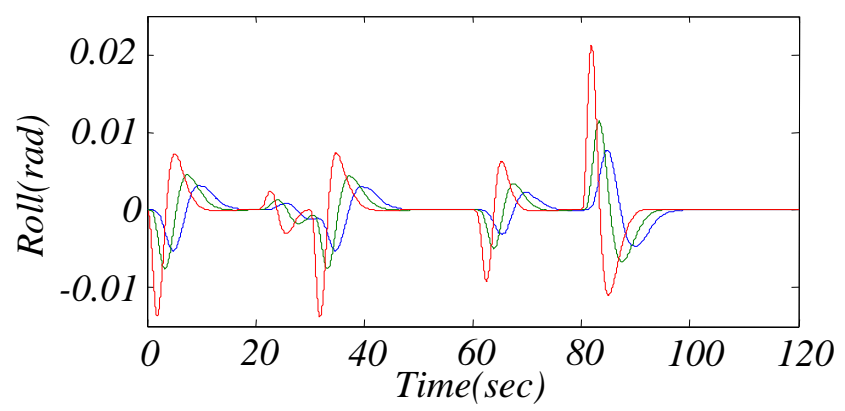

(e)

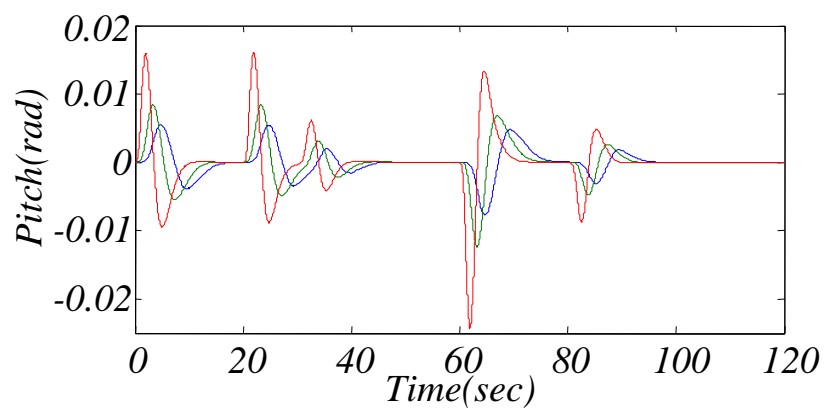

(f)

Figure.10 Trajectory of the output variables: (a) Trajectory of the yaw angle, (b) Trajectory of the $\mathrm{z}$ position, (c) Trajectory of the $x$ position, (d) Trajectory of the $y$ position, (e) Trajectory of the roll angle and (f) Trajectory of the pitch angle. 


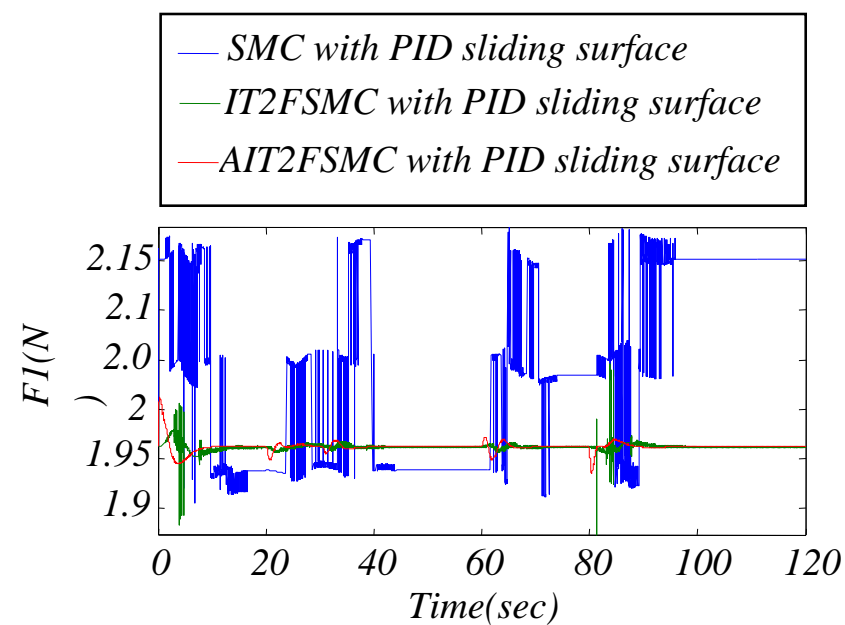

(a)

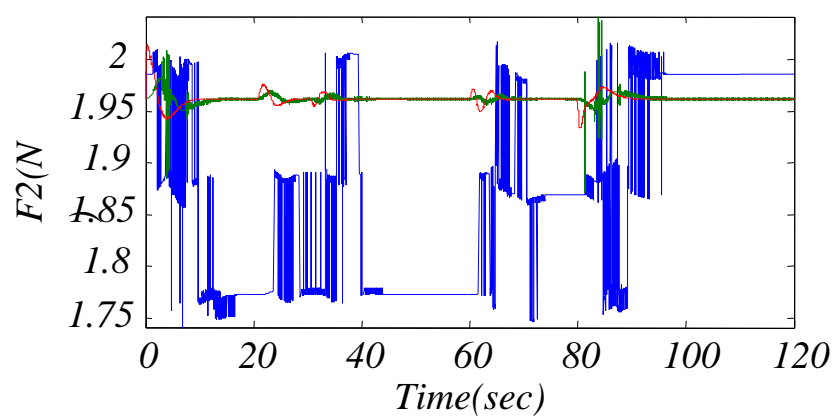

(b)

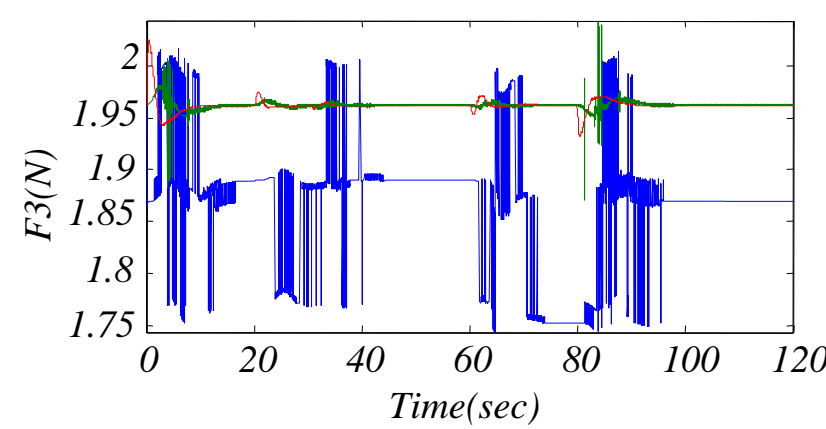

(c)

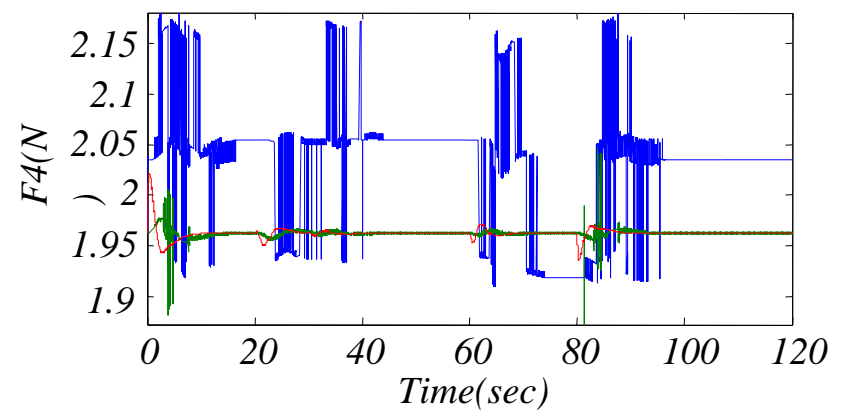

(d)

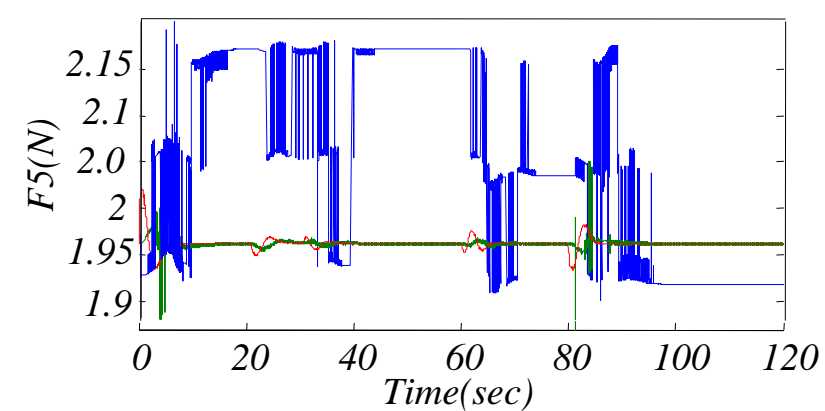

(e)

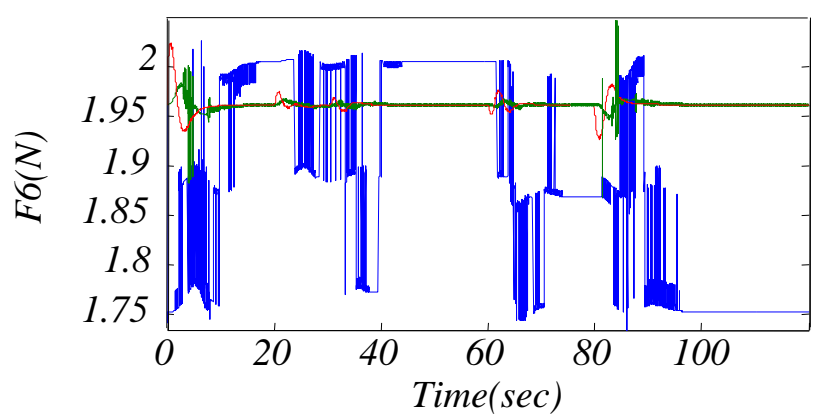

(f)

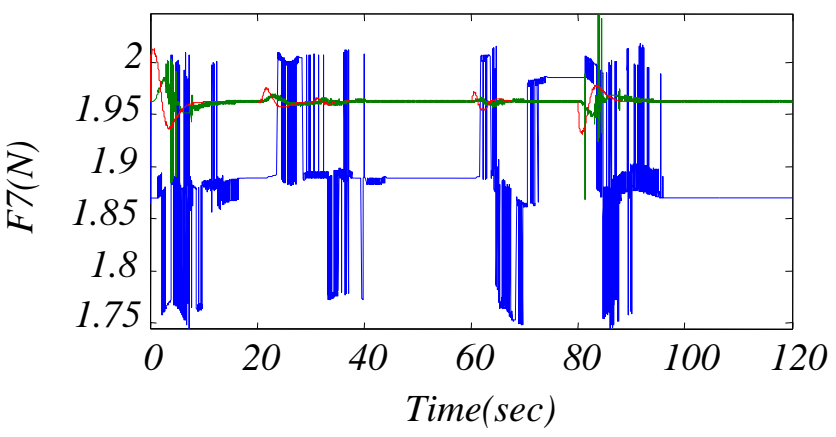

(g)

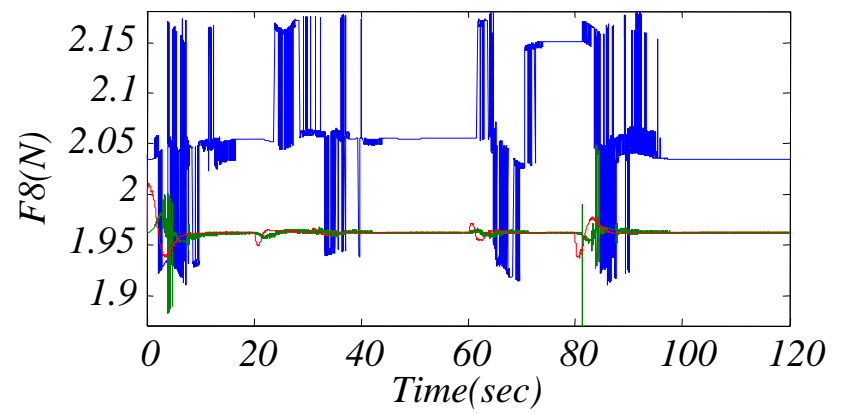

(h)

Figure.11 Control inputs: (a) Control input $F_{1}$, (b) Control input $F_{2}$, (c) Control input $F_{3}$, (d) Control input $F_{4}$, (e) Control input $F_{5}$, (f) Control input $F_{6}$, (g) Control input $F_{7}$, (h) Control input $F_{8}$. 
Table 3. Performance comparison of the controllers

\begin{tabular}{|l|c|c|c|c|c|c|}
\hline \multirow{2}{*}{ Algorithm } & \multicolumn{7}{|c|}{ IAE } \\
\cline { 2 - 7 } & $x$ & $y$ & $z$ & $\varphi$ & $\theta$ & $\psi$ \\
\hline $\begin{array}{l}\text { Conventional } \\
\text { SMC }\end{array}$ & 3.96 & 4.74 & 0.36 & 0.56 & 0.66 & 0.45 \\
\hline IT2FSMC & 2.61 & 3.12 & 0.24 & 0.37 & 0.44 & 0.3 \\
\hline AIT2FSMC & 1.62 & 1.84 & 0.012 & 0.21 & 0.23 & 0.01 \\
\hline \multirow{7}{*}{ Algorithm } & \multicolumn{7}{|c|}{ ITAE } \\
\cline { 2 - 8 } & $x$ & $y$ & $z$ & $\varphi$ & $\theta$ & $\psi$ \\
\hline $\begin{array}{l}\text { Conventional } \\
\text { SMC }\end{array}$ & 5.66 & 6.77 & 0.51 & 0.8 & 0.94 & 0.64 \\
\hline IT2FSMC & 0.77 & 0.92 & 0.07 & 0.11 & 0.13 & 0.08 \\
\hline AIT2FSMC & 0.53 & 0.60 & 0.003 & 0.06 & 0.05 & 0.002 \\
\hline
\end{tabular}

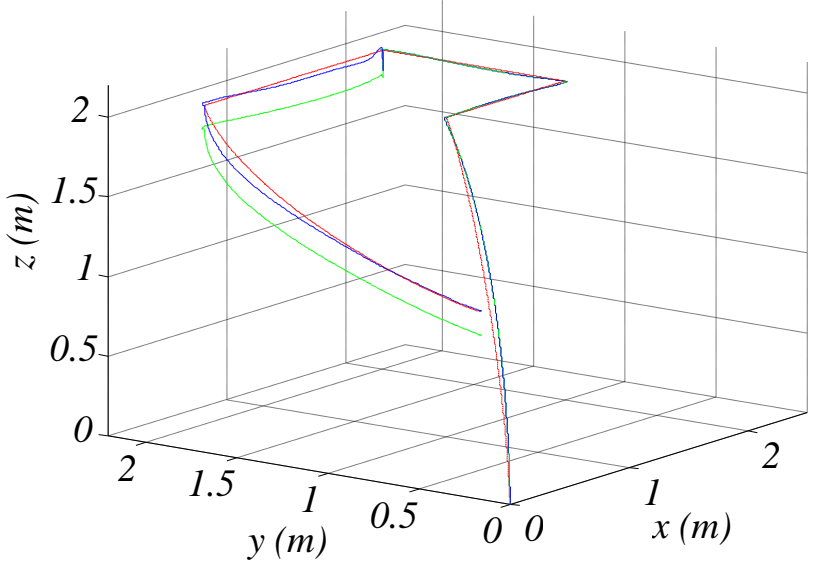

\begin{tabular}{|l|} 
Desired trajectory \\
Second order sliding mode control \\
Proposed AIT2FSMC
\end{tabular}

Figure.12 Comparison between the proposed controller and second order sliding mode control proposed in [12].

The efficiency of the proposed control method is illustrated in Fig. 12 via the comparison between the absolute position obtained using the proposed controller and the second order sliding mode controller presented in [12] in the presence of inertias and masse variations. For the comparison with the second order sliding mode controller presented by En-Hui Zheng et al. in [12]. The simulation results shown in Fig. 12 clearly demonstrate better performances: faster convergence, high-precision tracking and robustness with respect to the second order sliding mode controller presented in [12].

\section{Conclusions}

In this paper, the robust adaptive controller that is based on type-2 fuzzy inference systems and sliding mode control has been investigated. In this controller, the control law ensures the convergence of tracking errors and all signal plants in presence the parametric variations. This law incorporates adaptive parameters to compensate the parametric variations. The application of the developed method is carried out for a coaxial octorotor. The obtained simulation results show that this robust adaptive interval type-2 fuzzy sliding mode controller law maintains the tracking errors in an acceptable interval in the presence of extreme or significant parameter variations. In addition the chattering phenomenon that frequently appears in the conventional SMC is also eliminated without deteriorating the system robustness. In addition the comparative study performed with other works developed in the literature, has shown the effectiveness of the proposed control approach. In the future work the experimental implementation of the proposed control scheme will be addressed.

\section{References}

[1] G. Bo, L Xin, Z. Hui and W. Ling, "Quadrotor helicopter Attitude Control Using Cascade PID",In: Proc. of International Conf. On Control and Decision, Yinchuan, China, pp.5158-5163, 2016.

[2] X. Lw, D. Zhao and Y. Wu, "Application of Improved PSO in PID Parameter Optimization of Quadrotor",In: Proc. of International Conf. On Wavelet Active Media Technology and Information Processing, Chengdu, China, pp. 443-447, 2015.

[3] C. Liu, J. Pan and Y.Chang, "PID and LQR Trajectory Tracking Control for an Unmanned Quadrotor Helicopter: Experimental Studies", In: Proc. of Chinese Conf. On Control, Chengdu, China, pp. 10845-10850, 2016.

[4] V. Utkin, Sliding Modes in Control and Optimization, Springer-Verlag, 1992.

[5] M. Shaik and J. Whidborne, "Robust Sliding Mode Control of a Quadrotor",In: Proc. of International Conf. On control, Belfast, Ukraine, pp. 1-6, 2016.

[6] J. Xiong and G. Zhang, "Sliding Mode Control for a Quadrotor UAV with Parameter Uncertainties",In: Proc. of International Conf. On Control, Automation and Robotics, Hong Kong, Hong Kong, pp. 207-212, 2016.

[7] X. Rong, U. Ozguner, "Sliding mode control of a class of underactuated systems", Automatica, vol. 44, pp. 233-241, 2008.

[8] Z. Fang, Z. Zhi, L. Jun, W. Jian, "Feedback linearization and continuous sliding mode control for a quadrotor UAV",In: Proc of the IEEE Chinese Control Conference, Kunming, Yunnan, China, pp. 349-353, 2008. 
[9] S. Nadda and A. Swarup, "Development of Backstepping Based Sliding Mode Control for a Quadrotor",In: Proc. of International Conf. On Signal Processing \& its Applications, Kuala Lumpur, Malaysia, pp. 10-13, 2014.

[10]Z. He and L. Zhao, "Robust Chattering Free Backstepping/Backstepping Sliding Mode Control for Quadrotor Hovering",In: Proc. of International Conf. On Information Technology, Networking, Electronic and Automation Control, Chongqing, China, pp. 616-620, 2016.

[11] S. Hosseini and Mohammad Y, "MIMO sliding mode and backstepping control for a quadrotor UAV", In: Proc. of Iranian Conf. On Electrical Engineering, Tehran, Iran, pp. 994-999, 2015.

[12] E. H. Zheng, J. J. Xiong, J. L. Luo, "Second order sliding mode control for a quadrotor UAV", ISA Transactions, vol. 53, pp. 13501356, 2014.

[13] S.Sudhir and A. Swarup, "Second Order Sliding Mode Control for Quadrotor",In: Proc. of International Conf. On Control, Measurement and Instrumentation, Kolkata, India, pp. 92-96, 2016.

[14] S. Bouabdallah, R. Siegwart, "Backstepping and sliding-mode techniques applied to an indoor micro quadrotor",In: Proc of the IEEE international conference on Robotics and Automation, Barcelona, Spain, pp. 2247-2252, 2005.

[15] H. Alwi, C. Edwards, "Fault tolerant control of an octorotor using LPV based sliding mode control allocation",In: Proc of the American Control Conference, pp. 6505-6510, 2013.

[16] A. Marks, J.F. Whidborne, I, "Yamamoto. Control allocation for fault tolerant control of a VTOL octorotor",In: Proce of the UKACC Conference on Control, pp. 357-362, 2012.

[17] T. Madani, A. Benallegue, "Backstepping sliding mode control applied to a miniature quadrotor flying robot",In: Proce of the IEEE international conference on Industrial Electronics, Paris, France, pp. 700-705, 2006.

[18] A. Amer, E. Sallam, W. Elawady, "Adaptive fuzzy sliding mode control using supervisory fuzzy control for 3 DOF planar robot manipulators", Applied Soft Computing, vol. 11, pp. 4943-4953, 2011.

[19] K. Shyu, P. Chu, L. Shang, "Control of rigid robot manipulators via combination of adaptive sliding mode control and compensated inverse dynamics approach", IEE Proc. Control Theory Appl, vol. 143, pp. 283-288, 1996.
[20]Q. Ha, "Robust sliding mode controller with fuzzy tuning. Electron", Lett, vol. 17, pp. 16261628, 1996.

[21] S. Majd, L. Benjamin, F. Isabelle, F. Clovis, S. Hassan, S. Guillaume, "Fault diagnosis and fault-tolerant control strategy for rotor failure in an octorotor", In: Proc of the IEEE International Conference on Robotics and Automation, pp. 5266-5271, 2015. 